\title{
Avaliação do efeito de extrato de farinha de alfarroba (Ceratonia siliqua $L$.) na estabilidade oxidativa e cor de hambúrgueres congelados
}

\section{Evaluation of the effect of extract of carob flour (Ceratonia siliqua $L$.) on oxidative stability and color of frozen hamburgers}

\author{
Claudia Severo Rosa ${ }^{1 *}$; Ernesto Kubota ${ }^{1}$; Marília Stein ${ }^{2}$; \\ Gabriela Predebon Nogara ${ }^{2}$; Maira Vizzoto ${ }^{2}$
}

Resumo

\begin{abstract}
A oxidação lipídica é uma reação importante que limita a vida de prateleira de vários alimentos, especialmente em carnes. Os compostos fenólicos podem ser extraídos de vegetais e plantas e atuam como antioxidantes naturais em alimentos. A vagem da alfarroba é rica em taninos condensados (16$20 \%$ ) que podem exercer proteção contra a oxidação lipídica. Este trabalho teve como objetivo obter o extrato de alfarroba testando dois solventes e avaliar o efeito do extrato obtido com melhor capacidade antioxidante na estabilidade oxidativa e cor dos hambúrgueres congelados. Os solventes usados na extração foram a acetona a 70\% e a água. Foram realizadas análises de composição centesimal (teor de umidade, carboidratos, proteínas, lipídios e cinzas), medida de cor e índice de TBARS (Thiobarbituric Acid Reactive Substances) nos hambúrgueres. O extrato obtido com acetona a $70 \%$ foi o que apresentou os melhores resultados. Os valores encontrados na composição centesimal dos hambúrgueres de carne bovina mostraram que os mesmos estão de acordo com o Regulamento Técnico de Identidade e Qualidade de Hambúrguer. Com relação à cor, os hambúrgueres T3, T4 e T5 apresentaram uma coloração mais escura. No período final de estocagem o valor de TBARS para os hambúrgueres adicionados de diferentes níveis de extrato hidroacetônico apresentaram valores que variaram de 0,137 a $0,253 \mathrm{mg}$ (MDA) $/ \mathrm{kg}$ de amostra, enquanto o controle apresentou valor de $2,12 \mathrm{mg}$ (MDA)/kg de amostra. Pode-se concluir que os extratos adicionados proporcionaram estabilidade oxidativa aos hambúrgueres.
\end{abstract}

Palavras-chave: Farinha, hambúrguer, oxidação, cor

\begin{abstract}
Lipid oxidation is an important reaction that limits the shelf life of various foods, especially meats. Phenolic compounds can be extracted from vegetables and plants act as natural antioxidants in foods. The carob pod is rich in condensed tannins (16-20\%) that can exert protection against lipid oxidation. This study aimed to obtain the extract of carob testing two solvents and evaluating the effect of the extract obtained with better antioxidant capacity and oxidative stability of frozen hamburgers color. The solvent used in extraction was $70 \%$ acetone and water. Analyzes were conducted of proximate composition (moisture content, carbohydrates, protein, fat and ash), color measurement and TBARS (Thiobarbituric Acid Reactive Substances) in burgers. The extract obtained with acetone $70 \%$ showed the best results. The values found in the proximate composition of the beef burgers showed that they
\end{abstract}

\footnotetext{
${ }^{1}$ Profs. do Programa de Pós-Graduação em Ciência e Tecnologia dos Alimentos, Universidade Federal de Santa Maria, UFSM, Santa Maria, RS. E-mail: claudiasr37@yahoo.com.br; ernehk2008@yahoo.com.br

${ }^{2}$ Discentes, UFSM, Santa Maria, RS.E-mail: mari_nstein@hotmail.com; gabi10.nog@hotmail.com; mairavizzoto@yahoo.com.br * Autor para correspondência
} 
comply with the Technical Regulation of Identity and Quality Burger. Regarding the color, burgers T3, $\mathrm{T} 4$ and T5 showed a dark. In the final period of storage the amount of TBARS to the burgers added different levels of hidroacetonic extract showed values ranging from the 0.137 to $0.253 \mathrm{mg}$ (MDA)/ $\mathrm{kg}$ of sample, whereas the control showed a value of $2.12 \mathrm{mg}$ (MDA)/ $\mathrm{kg}$ sample. It can be concluded that the extracts provided added stability to oxidative burgers.

Key words: Flour, hamburger, oxidation, color

\section{Introdução}

Em produtos cárneos, a oxidação lipídica é uma reação importante que limita a vida de prateleira, sendo um dos mecanismos primários da deterioração da qualidade. As alterações na qualidade podem ser percebidas pelas mudanças de sabor, de cor, de textura, de valor nutricional e pela produção de compostos potencialmente tóxicos. Dentre os fatores extrínsecos que contribuem para o desenvolvimento da oxidação lipídica em carnes estão as condições de processamento, como a moagem, tratamento térmico, aplicação de alta pressão, ingredientes e tipo de embalagem (MARIUTTI; BRAGAGNOLO, 2009).

Os antioxidantes estão presentes de forma natural ou intencional nas gorduras dos alimentos para retardar o aparecimento dos fenômenos de oxidação, mantendo intactas suas características sensoriais. Os antioxidantes que se adicionam aos alimentos não devem causar efeitos fisiológicos negativos, produzir cores, odores nem sabores anômalos. Devem ser lipossolúveis, resistentes aos tratamentos a que seja submetido o alimento, ativos em baixas temperaturas e econômicos (ORDÓÑEZ, 2005).

Os compostos fenólicos podem ser extraídos de vegetais e plantas. Muitas ervas e especiarias, utilizadas como condimentos em alguns pratos, são excelentes fontes de compostos fenólicos. Tais substâncias têm demonstrado alto potencial antioxidante, podendo ser usadas como conservantes naturais para alimentos (ZHENG; WANG, 2001). Os compostos fenólicos exibem grande quantidade de propriedades fisiológicas (como antialergênica, antiarteriogênica, anti-inflamatória, antimicrobiana, antitrombótica e vasodilatadora), mas o principal efeito dos compostos fenólicos tem sido atribuído à sua ação antioxidante em alimentos (BALASUNDRAM; SUNDRAM; SAMMAN, 2006).

A vagem da alfarroba é caracterizada por alta quantidade de açúcares (40-50\%) e taninos condensados (16-20\%) (MAKRIS; KEFALIS, 2004). A atividade antioxidante dos polifenóis da farinha de alfarroba foi demonstrada por Avallone et al. (1997) que ao determinarem o teor de polifenóis na farinha de alfarroba encontram 19,5 mg de equivalente grama de ácido gálico (GAE)/g de farinha, estando em concordância com o estudo de Kumazawa et al. (2002) que verificaram 19,2 mg GAE/g de farinha. A farinha de alfarroba é produzida retirando-se as sementes, e, em seguida, a vagem é torrada e moída (YOUSIF; ALGHZAWI, 2000). Entretanto o uso para alimentação humana é limitado por causa do alto teor de taninos causando excesso de adstringência. Este trabalho teve como objetivo extrair e avaliar o efeito dos extratos de alfarroba na estabilidade oxidativa e cor dos hambúrgueres bovinos congelados.

\section{Materiais e Métodos}

As análises foram realizadas no departamento de Tecnologia e Ciência dos Alimentos na Universidade Federal de Santa Maria. A farinha de alfarroba "light" com informação nutricional $(26,6 \%$ de açúcares, $7,0 \%$ de fibra, $3 \%$ de proteína e $6,0 \%$ de gordura) foi adquirida em loja especializada de Porto Alegre - RS. Devido ao seu alto teor de açúcar, a farinha foi submetida à lavagem para a obtenção do extrato.

$\mathrm{O}$ açúcar da farinha foi removido com duas lavagens de água gelada a $7{ }^{\circ} \mathrm{C}$ por $24 \mathrm{~h}$ sob refrigeração na proporção de 1 parte de farinha 
para 5 partes de água gelada, após foi centrifugado por $10 \mathrm{~min}$ a $5000 \mathrm{rpm}$ e seco em estufa com ventilação (De Leo, Porto Alegre - RS) à $50{ }^{\circ} \mathrm{C}$ por 36 h (KUMAZAWA et al., 2002). A determinação de açúcares na farinha antes e após a lavagem foi realizada de acordo com o IAL (1985).

\section{Obtenção do extrato}

Para a extração dos compostos fenólicos foram utilizados com solventes a acetona a 70\% e a água. A extração com acetona a 70\% seguiu a metodologia descrita por Avallone et al. (1997). Dez gramas de farinha foram homogeneizadas com $100 \mathrm{~mL}$ de acetona a $70 \%$ por $5 \mathrm{~min}$, centrifugado por $10 \mathrm{~min}$ a $5000 \mathrm{rpm}$ e o sobrenadante coletado, após o solvente foi totalmente evaporado em rotaevaporador (Fisaton 802) com vácuo de $-760 \mathrm{mmHg}$ e temperatura da água do banho de $50{ }^{\circ} \mathrm{C}$. Na extração com água, $10 \mathrm{~g}$ de farinha foram homogeneizadas com 100 $\mathrm{mL}$ de água destilada por $5 \mathrm{~min}$, centrifugado por $10 \mathrm{~min}$ a $5000 \mathrm{rpm}$ e o sobrenadante coletado. Os extratos hidrocetônico e aquoso foram coletados em frasco de vidro âmbar ao abrigo da luz e mantidos a quatro ${ }^{\circ} \mathrm{C}$ até o momento de serem utilizados para a determinação de compostos fenólicos totais e atividade antioxidante.

\section{Quantificação dos fenólicos totais dos extratos}

Para a determinação de fenólicos totais utilizouse o método de Folin-Ciocalteu descrito por Singleton, Orthofer e Lamuela-Raventos (1999) e adaptado por Pereira (2009). Uma alíquota $(0,5$ $\mathrm{mL}$ ) da solução estoque foi misturada a $2,5 \mathrm{~mL}$ de reagente de Folin-Ciocalteu 0,2 $\mathrm{N}$ e, após $5 \mathrm{~min}$, adicionou-se $2 \mathrm{~mL}$ de solução de carbonato de sódio $\left(\mathrm{Na}_{2} \mathrm{CO}_{3}\right) 7,5 \%$. Após a incubação a temperatura ambiente $\left(25^{\circ} \mathrm{C}\right)$ por $2 \mathrm{~h}$, a absorbância foi medida a $760 \mathrm{~nm}$ em espectrofotômetro e comparada com a curva padrão de ácido gálico (faixa de $0-50 \mathrm{mg} / \mathrm{L}$ ): $\mathrm{Y}=0,0112 \mathrm{x}+0,0409$, onde $\mathrm{Y}$ é a absorbância e $\mathrm{x}$ é a concentração; $R^{2}=0,9871$. Os resultados foram expressos como mg equivalente de ácido gálico por litro de extrato (mg GAE/L). As análises foram realizadas em triplicata.

Determinação da atividade antioxidante do extrato

A atividade antioxidante foi determinada pela capacidade de sequestrar DPPH (2,2-difenil 1-picril-hidrazil) descrita por Brand-Williams et al. (1995) com adaptações. Consistiu na incubação por 30 min., de $500 \mu \mathrm{L}$ de uma solução etanólica de DPPH 0,1 mM com $500 \mu \mathrm{L}$ de soluções contendo concentrações crescentes de extrato de alfarroba $(0,02 ; 0,04 ; 0,09 ; 0,2 ; 0,3 ; 0,6 ; 1,25 ; 2,5$ e 5,0 , $10,15 \mathrm{mg} / \mathrm{mL}^{-1}$ ) em etanol $80 \%$ e aquoso, sendo a leitura realizada a $517 \mathrm{~nm}$. A solução "controle" consistiu de DPPH 0,1 mM em etanol. O percentual de sequestro do radical DPPH foi calculado em termos de percentagem, expresso como atividade antioxidante (AA\%) e calculada conforme a equação 1 :

$\mathrm{AA} \%=100-\{[($ Abs. amostra - Abs. branco $) \mathrm{x}$ $100] \div$ Abs. controle\}. Equação (1)

A determinação do $\mathrm{IC}_{50}$, foi obtida por regressão linear dos pontos plotados graficamente conforme (CARBONARI, 2005). Para a plotagem dos pontos, foram utilizados os valores das médias obtidas das triplicatas realizadas para cada um dos testes. O poder antioxidante do extrato de alfarroba foi calculado de acordo com a equação da curva da atividade antioxidante, $\mathrm{Y}=13,709 \mathrm{x}+2,3789$, onde Y é substituído por 50 e x representa o valor do $\mathrm{IC}_{50}$.

\section{Elaboração dos hambúrgueres}

Os hambúrgueres foram preparados com a mesma formulação básica: dianteiro bovino $75,0 \%$, gordura bovina 15,0\%, PTS (proteína texturizada de soja) 2,0\%, água/gelo 3,0\%, cloreto de sódio $2,0 \%$ e cebola/alho 3,0\% variando apenas os antioxidantes adicionados (TORRES et al., 1998). O extrato de alfarroba utilizado foi o hidroacetônico por ter 
apresentado os melhores resultados para compostos fenólicos totais e capacidade antioxidante. Elaborouse uma formulação controle, uma com $0,1 \%$ de antioxidante sintético BHT e quatro formulações de hambúrgueres com adição de extrato hidroacetônico de alfarroba $(0,5 ; 1,0 ; 1,5$ e $2,5 \%)$.

A trituração da carne bovina e da gordura foram realizadas utilizando um moedor elétrico (Jamar PJ22, São Paulo) provido de disco com aberturas de $5 \mathrm{~mm}$ de diâmetro. Após a moagem, a matériaprima foi misturada juntamente com a água e o cloreto de sódio durante 3 minutos para a extração das proteínas miofibrilares. Adicionou-se à massa cárnea: cebola desidratada, PTS, antioxidante BHT e o extrato de alfarroba. Após foram moldados hambúrgueres de $30 \mathrm{~g}$, embalados em sacos de polietileno e congelados a $-18{ }^{\circ} \mathrm{C}$ por até 150 dias.

\section{Determinação da composição química}

Os teores de umidade, proteína, gordura e cinzas foram determinados no quinto dia após a elaboração dos hambúrgueres, segundo o IAL (1985).

Determinação do índice de oxidação lipídica (TBARS)

As substancias reativas ao ácido tiobarbitúrico (TBARS) resultantes da oxidação lipídica das amostras de hambúrgueres foram determinadas nos dias 1, 30, 60, 90, 120 e 150 de armazenamento congelado, segundo o método de Raharjo, Sofos e Schmidt (1992), com modificações conforme (PEREIRA, 2009). Os tubos com as amostras e os reativos foram colocados em banho-maria fervente por 5 min., resfriados e a leitura realizada em espectrofotômetro a $531 \mathrm{~nm}$. A densidade ótica foi lida e multiplicada por 7,8. O resultado foi expresso em mg de malonaldeído (MDA)/Kg de amostra.

\section{Determinação de cor}

A determinação da cor foi realizada utilizandose o sistema CIE em aparelho Chroma Meter CR-300 (Minolta, Japão), através da leitura dos parâmetros $L^{*}$ (que representa a porcentagem de luminosidade, onde preto $0 \%$ e branco $100 \%$ ), $a^{*}$ (onde $+a^{*}$ vermelho) e $b^{*}$ (onde $+b^{*}$ amarelo). O valor de $\mathrm{h}^{*}$ (ângulo de cor) foi obtido por cálculo. As leituras foram realizadas a temperatura ambiente na superfície das amostras de carne bovina, com cinco repetições para cada amostra avaliada.

\section{Análise estatística}

$\mathrm{O}$ desenho experimental foi inteiramente casualizado, com um controle e 5 tratamentos, totalizando 6 unidades experimentais. Todos dados foram avaliados através de análise de variância (ANOVA) e as médias comparadas pelo teste de Tukey, considerando o nível de significância de 5\% (COSTA NETO, 1977).

\section{Resultados e Discussões}

Verificou-se que o açúcar presente na farinha de alfarroba (26,6\%) foi totalmente removido com duas lavagens sucessivas com água gelada. Resultados semelhantes foram obtidos por Kumazawa et al. (2002) trabalhando com farinha de alfarroba com 45,0\% de açúcar.

Os resultados na Tabela 1 mostram que a acetona a $70 \%$ extraiu maiores quantidade de compostos fenólicos totais $(7,36 \mathrm{mg} \mathrm{GAE} / \mathrm{mL})$, apresentando diferença significativa $(p<0,05)$ em relação a extração com água. 
Tabela 1.Valores médios de compostos fenólicos totais e $\mathrm{IC}_{50}$ dos extratos de alfarroba obtidos com acetona a $70 \% \mathrm{e}$ água.

\begin{tabular}{ccc}
\hline Extratos & Compostos fenólicos $(\mathrm{mg} \mathrm{GAE} / \mathrm{mL})$ & $\mathrm{IC}_{50}(\mathrm{mg} / \mathrm{mL})$ \\
\hline Extrato acetona $70 \%$ & $7,36^{\mathrm{a}} \pm 1,38$ & $3,47^{\mathrm{b}} \pm 2,65$ \\
Extrato aquoso & $2,12^{\mathrm{b}} \pm 1,89$ & $6,12^{\mathrm{a}} \pm 2,29$ \\
\hline
\end{tabular}

Médias com letras diferentes na mesma coluna são significativamente diferentes pelo teste de Tukey $(p<0,05)$; GAE: equivalente em ácido gálico.

Fonte: Elaboração dos autores.

Não existe sistema de extração com solventes que seja satisfatório para o isolamento de todos ou de classe específica de antioxidantes naturais, devido a diversos fatores. A natureza química desses compostos nos alimentos varia do simples ao altamente polarizado, há grande variedade e diferentes quantidades de compostos bioativos nos vegetais como os ácidos fenólicos, antocianinas e taninos (ANDREO; JORGE, 2006). Makris e Kefalas (2004) testaram a extração dos compostos fenólicos totais da alfarroba com vários solventes e verificaram que a extração com acetona a $80 \%$ foi a melhor (9,28 mg GAE/g) e com metanol o menos eficiente (2,20 mg GAE/g), já Avallone et al. (1997) testando a extração dos fenólicos da alfarroba com acetona a $100 \%$, acetona a $70 \%$ e metanol a $70 \%$, verificaram que a extração com acetona a $70 \%$ foi significativamente superior $(19,5 \mathrm{mg} \mathrm{GAE} / \mathrm{g}$ de farinha) aos outros solventes testados, resultados que reforçam a afirmação de Andreo e Jorge (2006) que os compostos fenólicos dependem da polaridade do solvente de extração. Vários fatores podem interferir no conteúdo de compostos fenólicos dos extratos, entre eles as condições de crescimento da planta, do solo, da preparação da planta para a extração, do processo de extração e da metodologia in vitro utilizada para identificar o conteúdo desses compostos (MADSEN; BERTELSEN, 1995).
O $\mathrm{IC}_{50}$ é um parâmetro que pode ser usado para determinar o potencial antioxidante, ele demonstra a quantidade necessária da planta ou substância para reduzir em 50\% o DPPH, simulando assim como atuará em um radical livre no organismo. Quanto maior o $\mathrm{IC}_{50}$, maior será a quantidade necessária de substância para exercer a atividade antioxidante, dessa forma, um baixo $\mathrm{IC}_{50}$ significa que a planta tem um grande poder antioxidante (NEGRI, 2007). Os resultados obtidos para a atividade antioxidante utilizando-se o método de DPPH mostram que dos extratos avaliados o extrato de alfarroba obtido com acetona a $70 \%$ (Tabela 1) foi o que apresentou menor $\mathrm{IC}_{50}(3,47 \mathrm{mg} / \mathrm{ml})$. Morais et al. (2009) avaliaram a atividade antioxidante de chás e condimentos mais consumidos no Brasil e verificaram valores de $\mathrm{IC}_{50}$ para boldo, orégano, chá preto, louro, e cravo de $2,08,1,08,0,96,0,76$ e $0,46 \mathrm{mg} / \mathrm{ml}$, respectivamente.

Observa-se na Tabela 2 que os teores de umidade, lipídios e cinzas não apresentam diferença significativa ao nível de $5 \%$ entre os tratamentos, mas o tratamento $\mathrm{T} 1$ diferiu estatisticamente dos demais apresentando maior teor de proteína, o que pode ter sido provocado pela falta de homogeneização da amostra. Os valores de gordura e proteína estão dentro dos estabelecidos pelo Regulamento Técnico de Identidade e Qualidade de Hambúrguer (BRASIL, 2000), que estabelece como valor máximo para gordura $23,0 \%$ e como valor mínimo para proteína $15,0 \%$. 
Tabela 2. Composição centesimal dos hambúrgueres bovinos congelados.

\begin{tabular}{llllll}
\hline Tratamento & Umidade (\%) & Lipídios (\%) & Proteínas (\%) & Cinzas (\%) & CHO (\%) \\
\hline Controle & $65,25^{\mathrm{a}} \pm 0,30$ & $10,60^{\mathrm{a}} \pm 0,54$ & $18,60^{\mathrm{a}} \pm 0,10$ & $2,33^{\mathrm{a}} \pm 0,13$ & $3,22^{\mathrm{a}} \pm 0,16$ \\
T1 & $65,26^{\mathrm{a}} \pm 0,28$ & $10,31^{\mathrm{a}} \pm 0,31$ & $19,90^{\mathrm{a}} \pm 0,10$ & $2,45^{\mathrm{a}} \pm 0,05$ & $2,08^{\mathrm{b}} \pm 0,13$ \\
T2 & $65,76^{\mathrm{a}} \pm 0,15$ & $10,21^{\mathrm{a}} \pm 0,37$ & $18,74^{\mathrm{a}} \pm 0,09$ & $2,61^{\mathrm{a}} \pm 0,20$ & $2,68^{\mathrm{a}} \pm 0,17$ \\
T3 & $65,52^{\mathrm{a}} \pm 0,08$ & $10,31^{\mathrm{a}} \pm 0,33$ & $18,69^{\mathrm{a}} \pm 0,25$ & $2,44^{\mathrm{a}} \pm 0,12$ & $3,04^{\mathrm{a}} \pm 0,11$ \\
T4 & $65,77^{\mathrm{a}} \pm 0,09$ & $10,10^{\mathrm{a}} \pm 0,23$ & $18,62^{\mathrm{ab}} \pm 0,15$ & $2,46^{\mathrm{a}} \pm 0,08$ & $3,05^{\mathrm{a}} \pm 0,13$ \\
T5 & $65,40^{\mathrm{a}} \pm 0,10$ & $10,34^{\mathrm{a}} \pm 0,30$ & $18,69^{\mathrm{ab}} \pm 0,22$ & $2,42^{\mathrm{a}} \pm 0,49$ & $3,15^{\mathrm{a}} \pm 0,09$ \\
\hline
\end{tabular}

Letras minúsculas iguais na mesma coluna não diferem estatisticamente ao nível de $5 \%$ pelo teste de Tukey. Controle: hambúrguer elaborado sem antioxidante, T1: hambúrguer elaborado com $0,1 \%$ de BHT, T2: hambúrguer elaborado com $0,5 \%$ de extrato de alfarroba, T3: hambúrguer elaborado com 1,0\% de extrato de alfarroba, T4: hambúrguer elaborado com 1,5\% de extrato de alfarroba e T5: hambúrguer elaborado com $2,5 \%$ de extrato de alfarroba.

Fonte: Elaboração dos autores.

Os valores médios de TBARS estão apresentados na Tabela 3, observa-se que no quinto mês de armazenamento todas as amostras apresentaram níveis de TBARS de no máximo 0,253 mg (MDA)/ $\mathrm{kg}$ sem diferenças significativas entre si, mas diferindo estatisticamente do controle com valor de 2,12 mg (MDA)/kg. Segundo Torres e Okani (1997) valores de até $1,59 \mathrm{mg}$ de (MDA)/ $\mathrm{kg}$ na carne não são percebidos por análise sensorial e também não causam danos à saúde do consumidor, podese observar que em nenhuma das etapas avaliadas os hambúrgueres tratados alcançaram este valor. Entretanto, os hambúrgueres controle apresentaram valores superiores e provavelmente seriam percebidos sensorialmente pelo consumidor em relação ao odor e sabor de ranço. O tratamento com BHT foi o que apresentou melhor comportamento, pois apresentou os menores valores médios de TBARS em relação aos demais tratamentos e portanto, apresentou melhor estabilidade oxidativa do hambúrguer neste período. Os hambúrgueres adicionados de extratos de alfarroba independente da concentração de extrato utilizado foram diminuindo os valores de TBARS ao longo dos meses e todos ao final do armazenamento apresentaram baixos valores indicando o efeito positivo do extrato de alfarroba, e a estabilidade oxidativa dos hambúrgueres entre esses tratamentos nesses períodos. Este comportamento do extrato de alfarroba em relação aos valores de TBARS pode ter sido devido a farinha de alfarroba possuir taninos condensados com alto peso molecular, que contêm suficientes grupos hidroxila fenólica que permitem a formação de ligações estáveis com as proteínas, na forma não oxidada os taninos reagem com as proteínas através de pontes de hidrogênio ou ligações hidrofóbicas, formando complexos estáveis tanino-proteína (DESHPANDE; CHERIAN; SALUNKHE, 1986) e segundo Hagerman et al. (1998) este complexo consegue reter pelo menos a metade da atividade antioxidante do tanino livre. Outro aspecto que pode ter influenciado os resultados é o fato de que muitos fenólicos simples podem apresentar atividade próoxidante e os taninos condensados apresentam pouca ou nenhuma atividade pró-oxidante (HAGERMAN et al., 1998). Entretanto futuras pesquisas devem ser feitas utilizando o extrato de farinha de alfarroba em sistemas cárneos mais complexos, onde são usados outros tipos de gorduras e ou nitritos para avaliar o efeito desses taninos condensados (BASTIDA et al., 2009). 
Tabela 3.Valores médios de TBARS expressos em mg de malonaldeído (MDA)/ kg de amostra.

\begin{tabular}{lllllll}
\hline Trat. & Mês 0 & Mês 1 & Mês 2 & Mês 3 & Mês 4 & Mês 5 \\
\hline Cont. & $0,162^{\mathrm{dE}} \pm 0,16$ & $0,175^{\mathrm{cE}} \pm 0,05$ & $0,252^{\mathrm{dD}} 0,02$ & $0,580^{\mathrm{Bc}} \pm 0,05$ & $0,786^{\mathrm{aB}} \pm 0,01$ & $2,12^{\mathrm{aA}} \pm 0,12$ \\
T1 & $0,235^{\mathrm{dA}} \pm 0,03$ & $0,189^{\mathrm{cBC}} \pm 0,09$ & $0,226^{\mathrm{dA}} \pm 0,10$ & $0,199^{\mathrm{eB}} \pm 0,01$ & $0,193^{\mathrm{cB}} \pm 0,01$ & $0,180^{\mathrm{bC}} \pm 0,01$ \\
T2 & $0,363^{\mathrm{CB}^{\mathrm{B}}} \pm 0,01$ & $0,486^{\mathrm{bA}} \pm 0,01$ & $0,487^{\mathrm{abA}} \pm 0,03$ & $0,276^{\mathrm{dcC}} \pm 0,01$ & $0,230^{\mathrm{cCD}} \pm 0,01$ & $0,216^{\mathrm{bD}} \pm 0,01$ \\
T3 & $0,435^{\mathrm{bC}} \pm 0,04$ & $0,609^{\mathrm{aB}} \pm 0,01$ & $0,391^{\mathrm{cD}} \pm 0,01$ & $0,880^{\mathrm{aA}} \pm 0,01$ & $0,808^{\mathrm{aA}} \pm 0,01$ & $0,137^{\mathrm{bE}} \pm 0,02$ \\
T4 & $0,422^{\mathrm{bA}} \pm 0,12$ & $0,435^{\mathrm{bA}} \pm 0,04$ & $0,423^{\mathrm{bc}} \pm 0,01$ & $0,345^{\mathrm{dB}} \pm 0,05$ & $0,328^{\mathrm{bB}} \pm 0,04$ & $0,203^{\mathrm{bC}} \pm 0,01$ \\
T5 & $0,699^{\mathrm{aA}} \pm 0,02$ & $0,660^{\mathrm{aB}} \pm 0,21$ & $0,490^{\mathrm{aC}} \pm 0,01$ & $0,434^{\mathrm{cD}} \pm 0,03$ & $0,373^{\mathrm{bE}} \pm 0,19$ & $0,253^{\mathrm{bF}} \pm 0,01$ \\
\hline
\end{tabular}

Letras minúsculas iguais na mesma coluna e letras maiúsculas na mesma linha não diferem estatisticamente ao nível de 5\% pelo teste de Tukey. Controle: hambúrguer elaborado sem antioxidante, T1: hambúrguer elaborado com 0,1\% de BHT, T2: hambúrguer elaborado com $0,5 \%$ de extrato de alfarroba, T3: hambúrguer elaborado com 1,0\% de extrato de alfarroba, T4: hambúrguer elaborado com 1,5\% de extrato de alfarroba e T5: hambúrguer elaborado com 2,5\% de extrato de alfarroba.

Fonte: Elaboração dos autores.

Bastida et al. (2009) adicionaram extratos de farinha de alfarroba comercias "Exxenterol e Liposterine" em sistemas cárneos na proporção de 30 $\mathrm{g} / \mathrm{kg}$ de carne e avaliaram a oxidação lipídica destes sistemas refrigerados e congelados. Nos sistemas cárneos congelados os resultados de TBARS foram superiores aos encontrados neste trabalho tendo a amostra controle atingido depois de seis meses 5,52 mg (MDA) $/ \mathrm{kg}$ de carne e os adicionados de extratos 0,95 e $0,75 \mathrm{mg}$ (MDA)/ $\mathrm{kg}$ de carne, porém o extrato de alfarroba proporcionou estabilidade oxidativa aos hambúrgueres.

A Tabela 4 apresenta os valores da determinação de cor dos hambúrgueres, pode-se notar que os valores de $\mathrm{L}^{*}$ diminuíram nos tratamentos ao longo dos 5 meses de armazenamento, com exceção do T1 (BHT) que não houve diferença significativa na cor. Este decréscimo provavelmente se deva a formação da cor escura em decorrência de reações de escurecimento (BOZKURT; BAYRAM, 2006). Segundo Ordóñez (2005) o processamento da carne altera consideravelmente a sua cor. O congelamento favorece a tonalidade pardacenta em face à dificuldade de penetração do $\mathrm{O}_{2}$ e por levar eletrólitos que favoreçem a metamioglobina.

Os hambúrgueres apresentaram uma tendência na redução da cor vermelha (parâmetro $a^{*}$ e $h^{*}$ ) durante os 5 meses de armazenamento, com poucas variações significativas entre os tratamentos, até o terceiro mês de armazenamento todos os hambúrgueres tiveram o mesmo comportamento não diferindo estatisticamente entre si, com exceção do tratamento com BHT, a partir do terceiro mês o hambúrguer com $1,5 \%$ de extrato apresentou uma cor menos vermelha, enquanto que os outros tratamentos com extrato de alfarroba apresentaram as mesmas tendências até o final do período de armazenamento. A redução no valor de $\mathrm{a}^{*}$ nos hambúrgueres tratados com extratos de alfarroba pode ser explicada pelo fato dos taninos presentes no extrato causarem descoloração possivelmente pela combinação com ferro da mioglobina retardando a oxidação lipídica (MITSUMOTO et al., 2005). 
Tabela 4. Médias dos parâmetros $\mathrm{L}^{*}, \mathrm{a}^{*}, \mathrm{~b}^{*} \mathrm{e} \mathrm{h}^{*}$ das amostras de hambúrgueres controle e dos adicionados de extrato bruto de alfarroba durante o período de armazenamento.

\begin{tabular}{|c|c|c|c|c|c|c|}
\hline \multirow[b]{2}{*}{ Trat. } & \multicolumn{6}{|c|}{ Tempo de armazenamento (meses) } \\
\hline & 0 & 1 & 2 & 3 & 4 & 5 \\
\hline & \multicolumn{6}{|c|}{$\mathrm{L}^{*}$} \\
\hline Cont. & $41,42^{\mathrm{a}} \pm 0,2$ & $41,59^{\mathrm{a}} \pm 0,4$ & $41,24^{\mathrm{ab}} \pm 0,24$ & $40,28^{\mathrm{ab}} \pm 0,3$ & $40,12^{\mathrm{b}} \pm 0,2$ & $37,72^{\mathrm{c}} \pm 0,38$ \\
\hline $\mathrm{T} 1$ & $42,55^{\mathrm{a}} \pm 0,46$ & $40,68^{\mathrm{a}} \pm 0,21$ & $41,98^{\mathrm{a}} \pm 0,17$ & $41,33^{\mathrm{a}} \pm 0,5$ & $41,28^{\mathrm{a}} \pm 0,39$ & $40,18^{\mathrm{a}} \pm 0,22$ \\
\hline $\mathrm{T} 2$ & $40,49^{\mathrm{a}} \pm 0,21$ & $39,35^{\mathrm{ab}} \pm 0,38$ & $40,20^{b} \pm 0,60$ & $38,49^{\mathrm{c}} \pm 0,49$ & $38,60^{\mathrm{c}} \pm 0,09$ & $37,57^{c} \pm 0,28$ \\
\hline $\mathrm{T} 3$ & $39,79^{\mathrm{a}} \pm 0,1$ & $40,63^{\mathrm{a}} \pm 0,41$ & $40,21^{\mathrm{ab}} \pm 0,58$ & $39,19^{b} \pm 0,62$ & $38,95^{\mathrm{b}} \pm 0,70$ & $38,74^{b} \pm 0,27$ \\
\hline $\mathrm{T} 4$ & $39,49^{\mathrm{a}} \pm 0,25$ & $40,31^{\mathrm{ab}} \pm 0,36$ & $40,15^{\mathrm{ab}} \pm 0,34$ & $38,75^{b} \pm 0,55$ & $38,85^{\mathrm{b}} \pm 0,26$ & $38,16^{\mathrm{c}} \pm 0,23$ \\
\hline \multirow[t]{2}{*}{ T5 } & $38,35^{\mathrm{b}} \pm 0,19$ & $38,50^{\mathrm{b}} \pm 0,32$ & $38,62^{b} \pm 0,26$ & $39,41^{\mathrm{a}} \pm 0,13$ & $37,94^{\mathrm{c}} \pm 0,26$ & $37,76^{c} \pm 0,21$ \\
\hline & \multicolumn{6}{|c|}{$\mathrm{a}^{*}$} \\
\hline Cont. & $22,83^{\mathrm{a}} \pm 0,79$ & $13,85^{\mathrm{b}} \pm 0,23$ & $14,15^{\mathrm{b}} \pm 0,15$ & $12,39^{\mathrm{b}} \pm 0,14$ & $11,26^{\mathrm{b}} \pm 0,37$ & $12,47^{\mathrm{b}} \pm 0,26$ \\
\hline $\mathrm{T} 1$ & $24,16^{\mathrm{a}} \pm 0,14$ & $19,22^{\mathrm{a}} \pm 0,33$ & $20,34^{\mathrm{a}} \pm 0,27$ & $20,10^{\mathrm{a}} \pm 0,14$ & $19,54^{a} \pm 0,21$ & $20,67^{a} \pm 0,15$ \\
\hline $\mathrm{T} 2$ & $20,56^{\mathrm{a}} \pm 0,16$ & $16,22 a^{b} \pm 0,2$ & $13,68^{\mathrm{b}} \pm 0,25$ & $12,52^{\mathrm{b}} \pm 0,11$ & $11,99^{\mathrm{b}} \pm 0,10$ & $12,47^{b} \pm 0,21$ \\
\hline $\mathrm{T} 3$ & $18,51^{\mathrm{a}} \pm 0,27$ & $13,57^{b} \pm 0,37$ & $13,16^{\mathrm{b}} \pm 0,27$ & $10,97^{b} \pm 0,11$ & $10,58^{\mathrm{b}} \pm 0,21$ & $10,13^{b} \pm 0,30$ \\
\hline $\mathrm{T} 4$ & $18,43^{\mathrm{a}} \pm 0,37$ & $13,87^{\mathrm{b}} \pm 0,22$ & $13,52^{\mathrm{b}} \pm 0,08$ & $10,90^{c} \pm 0,16$ & $10,44^{\mathrm{c}} \pm 0,10$ & $10,25^{\mathrm{c}} \pm 0,23$ \\
\hline \multirow[t]{2}{*}{$\mathrm{T} 5$} & $16,46^{\mathrm{a}} \pm 0,38$ & $11,86^{b} \pm 0,15$ & $11,92^{\mathrm{b}} \pm 0,11$ & $10,50^{\mathrm{b}} \pm 0,40$ & $9,64^{b} \pm 0,22$ & $9,89^{\mathrm{b}} \pm 0,38$ \\
\hline & \multicolumn{6}{|c|}{$\mathrm{b}^{*}$} \\
\hline Cont. & $11,49^{\mathrm{a}} \pm 0,29$ & $8,79^{c} \pm 0,40$ & $10,85^{\mathrm{a}} \pm 0,30$ & $9,70^{\mathrm{b}} \pm 0,24$ & $9,71^{\mathrm{b}} \pm 0,30$ & $9,59^{\mathrm{b}} \pm 0,40$ \\
\hline $\mathrm{T} 1$ & $12,77^{\mathrm{a}} \pm 0,21$ & $10,57^{\mathrm{a}} \pm 0,21$ & $11,54^{\mathrm{a}} \pm 0,15$ & $10,83^{\mathrm{a}} \pm 0,13$ & $10,05^{\mathrm{a}} \pm 0,21$ & $10,37^{\mathrm{a}} \pm 0,43$ \\
\hline $\mathrm{T} 2$ & $11,27^{\mathrm{a}} \pm 0,08$ & $10,09^{\mathrm{a}} \pm 0,17$ & $10,02^{\mathrm{a}} \pm 0,57$ & $8,94^{b} \pm 0,26$ & $9,50^{\mathrm{b}} \pm 0,31$ & $8,91^{\mathrm{b}} \pm 0,12$ \\
\hline $\mathrm{T} 3$ & $10,31^{\mathrm{a}} \pm 0,15$ & $9,73^{\mathrm{a}} \pm 0,23$ & $10,35^{\mathrm{a}} \pm 0,10$ & $10,02^{\mathrm{a}} \pm 0,13$ & $9,42^{\mathrm{ab}} \pm 0,26$ & $8,45^{\mathrm{b}} \pm 0,36$ \\
\hline $\mathrm{T} 4$ & $10,10^{\mathrm{a}} \pm 0,16$ & $9,66^{\mathrm{a}} \pm 0,09$ & $9,74^{\mathrm{a}} \pm 0,14$ & $8,82^{\mathrm{b}} \pm 0,16$ & $9,27^{\mathrm{a}} \pm 0,19$ & $9,60^{\mathrm{a}} \pm 0,14$ \\
\hline \multirow[t]{2}{*}{ T5 } & $9,63^{b} \pm 0,26$ & $9,04^{\text {bc }} \pm 0,09$ & $10,36^{\mathrm{a}} \pm 0,27$ & $10,41^{\mathrm{a}} \pm 0,18$ & $9,35^{b} \pm 0,10$ & $8,45^{\mathrm{c}} \pm 0,07$ \\
\hline & \multicolumn{6}{|c|}{$\mathrm{h}^{*}$} \\
\hline Cont. & $29,70^{\mathrm{c}} \pm 1,38$ & $35,99^{b} \pm 1,53$ & $39,40^{\mathrm{ab}} \pm 1,13$ & $42,29^{\mathrm{a}} \pm 0,44$ & $45,30^{\mathrm{a}} \pm 0,50$ & $41,46^{a} \pm 1,35$ \\
\hline $\mathrm{T} 1$ & $30,94^{\mathrm{a}} \pm 0,31$ & $31,81^{\mathrm{a}} \pm 0,80$ & $32,77^{\mathrm{a}} \pm 0,12$ & $30,58^{\mathrm{a}} \pm 0,93$ & $30,59^{\mathrm{a}} \pm 0,59$ & $29,59^{\mathrm{a}} \pm 0,09$ \\
\hline $\mathrm{T} 2$ & $31,93^{\mathrm{c}} \pm 0,28$ & $35,43^{\mathrm{ab}} \pm 0,59$ & $42,22^{\mathrm{a}} \pm 0,60$ & $39,49^{b} \pm 1,15$ & $45,32^{a} \pm 1,32$ & $39,49^{b} \pm 0,24$ \\
\hline $\mathrm{T} 3$ & $32,35^{\mathrm{c}} \pm 0,74$ & $39,60^{\mathrm{ab}} \pm 0,63$ & $42,42^{\mathrm{ab}} \pm 0,36$ & $47,25^{\mathrm{a}} \pm 0,68$ & $46,31^{\mathrm{a}} \pm 1,44$ & $44,25^{\mathrm{a}} \pm 1,17$ \\
\hline $\mathrm{T} 4$ & $31,92^{\mathrm{c}} \pm 0,58$ & $38,72^{\mathrm{b}} \pm 0,35$ & $45,51^{a} \pm 0,56$ & $43,31^{\mathrm{ab}} \pm 1,03$ & $46,20^{\mathrm{a}} \pm 0,40$ & $47,92^{\mathrm{a}} \pm 0,41$ \\
\hline T5 & $33,69^{\mathrm{c}} \pm 0,55$ & $41,48^{\mathrm{b}} \pm 0,60$ & $45,56^{\mathrm{ab}} \pm 1,11$ & $49,72^{\mathrm{a}} \pm 0,80$ & $50,36^{\mathrm{a}} \pm 0,70$ & $48,06^{\mathrm{a}} \pm 1,06$ \\
\hline
\end{tabular}

Letras minúsculas iguais na mesma linha não diferem estatisticamente ao nível de 5\% pelo teste de Tukey. Controle: hambúrguer elaborado sem antioxidante, T1: hambúrguer elaborado com $0,1 \%$ de BHT, T2: hambúrguer elaborado com $0,5 \%$ de extrato de alfarroba, T3: hambúrguer elaborado com $1,0 \%$ de extrato de alfarroba, T4: hambúrguer elaborado com 1,5\% de extrato de alfarroba e T5: hambúrguer elaborado com $2,5 \%$ de extrato de alfarroba.

Fonte: Elaboração dos autores.

\section{Conclusão}

O extrato de alfarroba obtido a partir da extração com acetona a $70 \%$ apresentou maior conteúdo de fenólicos e maior poder antioxidante.

Os níveis de TBARS mostram que os extratos de alfarroba adicionados aos hambúrgueres nas diferentes concentrações proporcionaram estabilidade oxidativa.

O parâmetro de cor $L^{*}$ diminuiu ao longo do armazenamento evidenciando que houve um escurecimento nos hambúrgueres. 


\section{Agradecimentos}

Os autores agradecem a FAPERGS pelo auxílio recebido.

\section{Referências}

ANDREO, D.; JORGE, N. Antioxidantes naturais: técnicas de extração. Boletim do Centro de Processamento de Alimentos, Curitiba, v. 24, n. 2, p. 319-336, 2006.

AVALLONE, R.; PLESSI, M.; BARALDI, M.; MONZANI, A. Determination of chemical composition of Carob (Ceratonia Siliqua): protein, fat, carbohydrates, and tannins. Journal of Food Composition and Analysis, New York, v. 10, n. 2, p.166-172, 1997.

BALASUNDRAM, N.; SUNDRAM, K.; SAMMAN, S. Phenolic compounds in plants and agri-industrial byproducts: antioxidant activity, occurrence, and potential uses. Food Chemistry, London, v. 99, n. 1, p. 191-203, 2006.

BASTIDA, S.; SÁNCHEZ-MUNIZ, F.; OLIVERO, R.; PÉRES-OLLEROS, L.; RUIZ-ROSO, B.; JIMENESCOLMENERO, F. Antioxidante activity of carob fruit extracts in cooked pork meat systems during chilled and frozen storage. Food Chemistry, London, v. 116, n. 3, p.748-754, 2009.

BOZKURT, H.; BAYRAM, M. Colour and textural attributes of sucuk during ripening. Meat Science, Barking, v. 73, n. 3, p. 344-350, 2006.

BRAND-WILLIANS, W.; CUVELIER, M. E.; BERSET, C. Use of a free radical method to evaluate antioxidant activity. Food Science and Technology, London, v. 28, n. 1, p. 25-30, 1995.

BRASIL. Ministério da Agricultura e do Abastecimento. Regulamento técnico da identidade e qualidade de hambúrguer. Instrução normativa $n^{\circ}$ 20, de 31/07/2000. Diário Oficial [da] República Federativa do Brasil, Brasília, 2000. p. 7-9.

CARBONARI, K. A. Avaliação do potencial antioxidante (In vitro e In vivo) e antiinflamatório de Ouratea parviflora, Polymnia sonchifolia e Marlierea obscura. 2005. Dissertação (Mestrado em Biotecnologia Aplicada à Saúde) - Universidade Federal de Santa Catarina, Florianópolis.

COSTA NETO, P. L. O. Estatística. São Paulo: Edgard Bluches, 1977. $264 \mathrm{p}$.

DESHPANDE, S. S.; CHERIAN, M.; SALUNKHE, D. Tannin analysis of food products. CRC Critical Reviews in Food Science and Nutrition, Boca Raton, v. 4, n. 4, p. 401-449, 1986.

HAGERMAN, A. N.; RIEDL, G.; JONES, A.; SOVIK, K. N.; RICHARD, N. T.; HARTZFELD, P. W.; RIECHEL, T. L. High molecular weight plant polyphenolics (tannins) as biological antioxidants. Journal of Agricultural and Food Chemistry, Easton, v. 46, n. 5, p. 1887-1892, 1998.

INSTITUTO ADOLFO LUTZ - IAL. Normas analíticas do Instituto Adolfo Lutz: métodos químicos e físicos para análise de alimentos. 2. ed. São Paulo: O Instituto, 1985.

KUMAZAWA, S., TANIGUCHI, M., SUZUKI, Y.; SHIMURA, M.; KWOU, M.; NAKAIAMA, T. Antioxidant activity of polyphenols in Carob pods. Journal of Agricultural and Food Chemistry, New York, v. 50, n. 2, p. 373-377, 2002.

MADSEN, H.; BERTELSEN, G. Spices as antioxidants. Trends in Food Science and Technology, Edinburgh, v. 6, n. 8, p. 271-277, 1995.

MAKRIS, D.; KEFALAS, P. Carob pods (Ceratonia siliqua L.) as a source of poliphenolic antioxidants. Food Technology and Biotechnology, USA, v. 42, n. 2, p. 105108, 2004.

MARIUTTI, L. R.; GRAGAGNOLO, N. A oxidação lipídica em carne de frango e o impacto da adição de sálvia (Salvia officinalis, L.) e de alho (Allium sativum, L.) como antioxidantes naturais. Revista do Instituto Adolfo Lutz, São Paulo, v. 68, n. 1, p. 1-11, 2009.

MITSUMOTO, M.; O'GRADY, M.; KERRI, J.; BUCKLEY, D. Addition of tea catechins and vitamin C on sensory evalution, colour and lipid stability during chillid storage in cooked, or raw beef and chicken patties. Meat Science, Barking, v. 69, n. 4, p. 773-779, 2005.

MORAIS, S.; CAVALCANTI, E.; COSTA, S.; AGUIAR, L. Ação antioxidante de chás e condimentos de grande consumo no Brasil. Revista Brasileira de Farmacognosia, São Paulo, v. 19, n. 1b, p. 315-320, 2009.

NEGRI, M. L. S. Secagem das folhas de EspinheiraSanta-Maytenus ilicifolia Mart. Ex Reiss. sob diferentes temperaturas e influência nos teores de polifenóis, na atividade antioxidante e nos aspectos microbiológicos. 2007. Dissertação (Mestrado em Ciências Farmacêuticas) - Universidade Federal do Paraná, Curitiba.

ORDÓÑEZ, J. A. Tecnologia de alimentos: componentes dos alimentos e processos. Porto Alegre: Artmed, 2005.

PEREIRA, M. G. Aplicação de antioxidantes naturais em carne mecanicamente separada (CMS) de ave. 2009. Dissertação (Mestrado em Ciência e Tecnologia de Alimentos) - Universidade Federal de Santa Maria, Santa Maria. 
RAHARJO, S.; SOFOS, N. J.; SCHMIDT, R. G. Improved speed, specificity, and limit of determination of an aqueous acid extraction thiobarbituric acid- $\mathrm{C}_{18}$ method for measuring lipid peroxidation in beef. Journal of Agricultural and Food Chemistry, Easton, v. 40, n. 11, p. 2182-2185, 1992.

SINGLETON, V. L.; ORTHOFER, R.; LAMUELARAVENTOS, R. M. Analysis of total phenols and oxidation substrates and antioxidants by means of FolinCiocalteau reagent. Methods in Enzymology, Pasadena, v. 299, n. 1, p. 152-178, 1999.

TORRES, E. A. F.; OKANI, E. T. Teste de TBA: ranço em alimentos. Revista Nacional da Carne, São Paulo, v. 243, n. 4, p. 68-76, 1997.
TORRES, F. S.; RIMOLI, C. D.; OLIVO, R.; HATANO, M.; SHIMOKOMAKI, M. Papel do sal iodado na oxidação lipídica em hambúrgueres bovino e suíno (misto) ou de frango. Ciência e Tecnologia de Alimentos, Campinas, v. 18, n. 1, p. 49-52, 1998.

YOUSIF, A. K.; ALGHZAWI, H. M. Processing and characterization of carob powder. Food Chemistry, London, v. 69, n. 3, p. 283-287, 2000.

ZHENG, W.; WANG, S. Antioxidant activity and phenolic composition in selected herbs. Journal of Agricultural and Food Chemistry, Easton, v. 49, n. 11, p. 5165-5170, 2001. 